\section{ORIGINAL RESEARCH}

\author{
T.A. Bley \\ M. Uhl \\ J. Carew \\ M. Markl
}

D. Schmidt

H.-H. Peter

M. Langer

O. Wieben

\title{
Diagnostic Value of High-Resolution MR Imaging in Giant Cell Arteritis
}

BACKGROUND AND PURPOSE: Clinical indications of giant cell arteritis may be unspecific, and noninvasive diagnosis is often difficult. This study investigated the hypothesis that high-resolution MR imaging of the superficial cranial arteries is a noninvasive imaging technique that can detect the occurrence of giant cell arteritis.

MATERIALS AND METHODS: Contrast-enhanced, high-resolution MR imaging was performed on 64 consecutive patients with suspected giant cell arteritis. Mural thickness, lumen diameter, and a mural contrast enhancement score were assessed with T1-weighted spin-echo images with submillimeter in-plane spatial resolution. The final rheumatologist's diagnosis according to the clinical criteria of the American College of Rheumatology including laboratory tests and results of temporal artery biopsies from 32 patients was used as a "gold standard" for the evaluation of the MR imaging findings.

RESULTS: All of the examinations provided diagnostic image quality. Evaluation of the mural inflammatory MR imaging signs for diagnosing vasculitis resulted in a sensitivity of $80.6 \%$ and a specificity of $97.0 \%$. In comparison, histology results alone showed a sensitivity of $77.8 \%$ and specificity of $100 \%$. The mean wall thickness increased significantly from $0.39 \mathrm{~mm}( \pm 0.18 \mathrm{~mm})$ to $0.74 \mathrm{~mm}( \pm 0.32$ $\mathrm{mm} ; P<.001)$, and the lumen diameter decreased significantly from $0.84 \mathrm{~mm}( \pm 0.29 \mathrm{~mm})$ to $0.65 \mathrm{~mm}$ $( \pm 0.38 \mathrm{~mm} ; P<.05)$ for patients with giant cell arteritis.

CONCLUSION: Contrast-enhanced, high-resolution MR imaging allows noninvasive assessment of mural inflammation in giant cell arteritis with good diagnostic certainty. Measures of mural thickening and contrast enhancement can be obtained in these small vessels and provide valuable vasculitic MR imaging findings.

G iant cell arteritis (GCA) is a chronic, granulomatous vasculitis $^{1}$ of large and medium sized arteries and is often associated with polymyalgia rheumatica. ${ }^{2}$ Clinical symptoms include new onset or new type of headache and tenderness of the temporal artery or decreased pulsatility of the temporal artery on palpation. Inflammatory involvement is not limited to the superficial temporal artery but may also include other cranial arteries, for example, the occipital arteries. ${ }^{3-5}$ Visual symptoms, such as diplopia and amaurosis fugax, may occur, with blindness being a dreaded complication. Laboratory findings typically reveal an inflammatory response with elevated erythrocyte sedimentation rate (ESR) and increased values of C-reactive protein (CRP). Criteria for classification of GCA were proposed by the American College of Rheumatology (ACR). ${ }^{6}$ In clinical routine, the correct diagnosis of GCA is often difficult. For reliable diagnosis, a temporal artery biopsy (TAB), which shows signs of vasculitis with mononucleated infiltrates of all mural layers or occurrence of giant cells, is usually required. ${ }^{7,8}$ Certainty about the correct diagnosis is needed, especially in view of the required long-term treatment with corticosteroids and their adverse affects. Characteristic findings of temporal arteritis can be visualized by color duplex ultrasonography, with a dark halo being the most specific sign.

Received November 18, 2006; accepted after revision March 27, 2007.

From the Departments of Diagnostic Radiology and Medical Physics (T.A.B., M.U., M.M., M.L.), Ophthalmology (D.S.), and Rheumatology and Clinical Immunology (H.-H.P.), University Hospital Freiburg, Freiburg, Germany; Departments of Biostatistics and Radiology (J.C.), Emory University, Atlanta, Ga.; and Departments of Medical Physics and Radiology (O.W.), University of Wisconsin-Madison, Madison, Wis.

Our research group was supported by Federal Ministry for Education and Research (BMBF) grant BMBF/KNR $01 \mathrm{Gl} / 9949$.

Please address correspondence to Thorsten A. Bley, University Hospital Freiburg, Department of Diagnostic Radiology and Medical Physics, Hugstetter Str 55, 79106 Freiburg, Germany; e-mail: thorsten.bley@uniklinik-freiburg.de

DOI 10.3174/ajnr.A0638
Its clinical value in the diagnosis of GCA, however, has been debated. ${ }^{2,7,9-11}$ Schmidt et al $^{7}$ reported that diagnosis of GCA can be derived without performing TAB in patients with typical clinical signs and a positive ultrasonography examination. On the other hand, Salvarani et $\mathrm{al}^{10}$ stated that ultrasonography is not better than a careful physical examination for the detection of biopsy-proved GCA.

MR imaging is an excellent tool for assessing the human anatomy and vasculature in a multitude of obliquities. It is a noninvasive and reproducible imaging technique that is routinely used by radiologists for the interpretation of vasculitic changes in larger vessels. With the use of gadolinium-based intravenous contrast agents, mural inflammatory changes because of vasculitis can be readily revealed as increased mural contrast enhancement. The technical advances in high-field MR imaging systems and coil design allow for high-resolution imaging with submillimeter resolution. ${ }^{12}$ Recently, a novel MR imaging protocol for vessel wall imaging of the temporal artery and for assessment of the cranial involvement pattern in patients with GCA was introduced. ${ }^{3,13}$ In this study, we tested the hypothesis that high-resolution MR imaging of the superficial cranial arteries can detect GCA in a larger patient population. The statistic assessment includes evaluation of the diagnostic impact of the different imaging findings (wall thickness, lumen diameter, and severity of mural contrast enhancement), comparisons with results from $\mathrm{TAB}$ alone, and comparisons with clinical diagnosis based on the criteria of the ACR, including biopsy, if applicable.

\section{Materials and Methods}

\section{Study Population}

Sixty-four consecutive patients (31 women and 33 men; age range, $44-85$ years; mean age, 68 years) referred to the Department of Ra- 
diology over a period of 18 months were included in the study. All of the patients were either suspected of having GCA or GCA was a potential differential diagnosis in the clinical presentation with localized headaches, tenderness of the superficial temporal arteries, visual impairments, and/or serologic signs of inflammation. The patients were referred for MR imaging by rheumatologists, ophthalmologists, and neurologists experienced in diagnosing and treating patients with GCA. Written informed consent was obtained from each patient before the MR imaging investigation. The study was approved by the local ethics review committee and follows the Declaration of Helsinki.

\section{Patients with GCA}

Within the patient population, 31 patients were found to be GCA positive. Unilateral TAB was performed in 27 of these 31 patients with true-positive findings in 21 patients and false-negative findings in 6 patients compared with the rheumatologist's final diagnosis according to the ACR clinical criteria including follow-up visits over at least 6 months after initial presentation. Of the 31 GCA-positive patients, 25 had started corticosteroid treatment 1-14 days (mean, 3 days) before the MR imaging examination. Two other patients had received long-term steroid treatment for 5 and 12 months, respectively, whereas 4 patients had not received any steroid treatment before the MR imaging examination.

\section{Control Patients}

The control group consisted of 33 patients in which GCA was ultimately ruled out by the ACR criteria. In this patient group, 17 subjects had received corticosteroid treatment before the MR examination (1-210 days; mean, 31 days), and a unilateral TAB was performed in 5 subjects.

\section{MR Imaging Examination}

High-resolution MR imaging was performed on a $1.5 \mathrm{~T}$ system in 29 patients and on a $3 \mathrm{~T}$ system in 38 patients (Siemens Magnetom Sonata and Trio, respectively; Siemens Medical Solutions, Erlangen, Germany). In both instances, a commercially available 8-element phased-array head coil was used. Postcontrast, fat-saturated multisection T1-weighted spin-echo (SE) images were acquired with a submillimeter spatial resolution of $195 \times 260 \mu \mathrm{m}$. For imaging at $1.5 \mathrm{~T}$, imaging parameters were as follows: TR/TE, $535 \mathrm{~ms} / 22 \mathrm{~ms}$; bandwidth, $65 \mathrm{~Hz} /$ pixel; FOV, $200 \times 200 \mathrm{~mm}^{2}$; acquisition matrix size, $1024 \times 768$; and number of acquisitions (averages), 1 . Total acquisition time for 10 sections was 6:55 minutes. At 3T, increased signal-tonoise ratio (SNR) was used for a slight increase in bandwidth (76 $\mathrm{Hz} /$ pixel) and thus reduction in a TR of $500 \mathrm{~ms}$. Images were acquired with FOV $=200 \times 200 \mathrm{~mm}^{2}$, and acquisition matrix size $=1024 \times$ 768. In addition, half Fourier encoding (half Fourier factor, 6/8) was used to decrease total scan time (10 sections) to 4:52 minutes. Three consecutive acquisitions (10 gapless sections; section thickness, 3 $\mathrm{mm}$ ) covered a volume that stretched over $90 \mathrm{~mm}$. No special shimming was performed. The acquisition of SE images was initiated approximately 1 minute after venous injection of $0.1 \mathrm{mmol} / \mathrm{kg}$ of a gadolinium-based contrast agent (Magnevist; Schering, Germany). In all but 1 case, MR imaging was performed before biopsy of the temporal artery.

\section{MR Imaging Evaluation}

All of the images were evaluated using state-of-the-art radiology work stations (J-Vision; Tiani, Vienna, Austria) in a consensus reading of 2 radiologists who were blinded to all of the other clinical data including biopsy results. The evaluability of the cranial arteries was re-
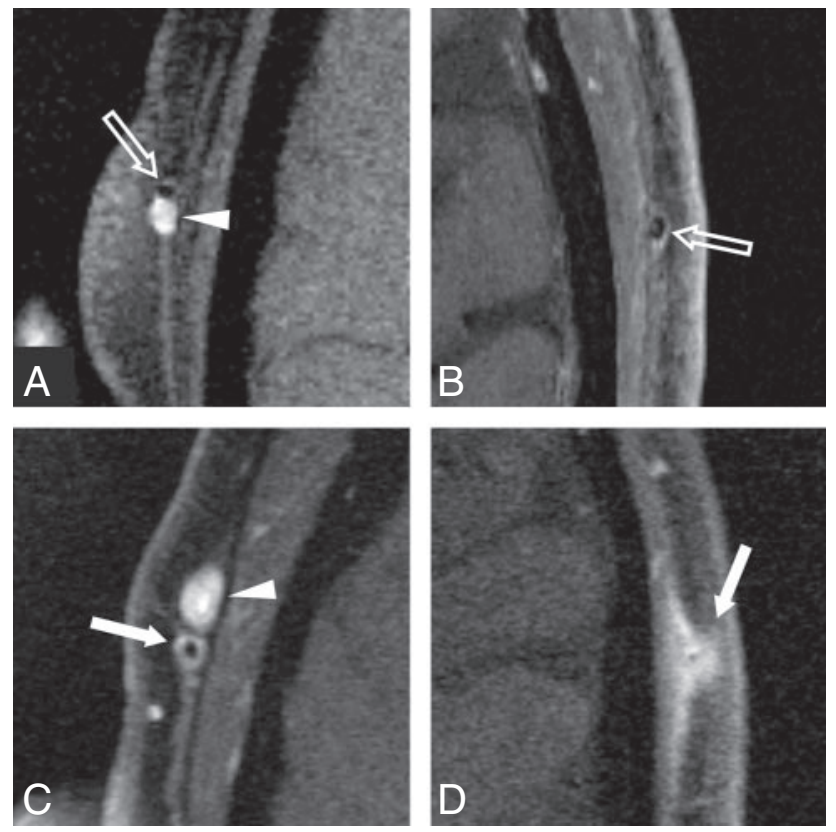

Fig 1. Enlargements of $3 T$ transversal postcontrast fat-suppressed T1-weighted SE image of the superficial temporal arteries of 4 different patients representing typical images of each grade of the 4-point ranking scale. Temporal artery biopsy is negative in cases $A$ and $B$, and suspected diagnosis of giant cell arteritis is validated by histology in cases $C$ and $D$. The concomitant veins (arrowheads in $A$ and $C$ ) display homogeneous signal intensity increase because of low venous flow. $A$, Mural thickness $<0.5 \mathrm{~mm}$ and no mural enhancement; rating " 0 ." Note the intraluminal signal intensity void (light arrow) because of arterial flow. $B$, Mural thickness $<0.5 \mathrm{~mm}$ with only slight contrast enhancement (light arrow), probably because of enhancing vasa vasorum; rating "1." $C$, Mural thickening $>0.6$ $\mathrm{mm}$ and prominent mural enhancement (arrow); rating " 2 ." $D$, Strong mural thickening $>0.7$ $\mathrm{mm}$ and strong mural enhancement (arrow); rating "3." The arterial lumen is still patent, as signal intensity void consistent with flow can be seen.

corded. For the MR imaging score of mural inflammation, a previously proposed 4-point ranking scale was used: 0, no mural thickening $(<0.5 \mathrm{~mm})$ and no mural enhancement; 1 , no mural thickening $(<0.5 \mathrm{~mm})$ with only slight contrast enhancement; 2 , mural thickening $(>0.6 \mathrm{~mm})$ and prominent mural enhancement; and 3, strong mural thickening $(>0.7 \mathrm{~mm})$ and strong mural enhancement. Scores of 0 and 1 were considered as physiologically normal, whereas 2 and 3 were considered as signs of mural inflammation (Fig 1). Luminal diameter and wall thickness were measured electronically on enlarged images using the radiology workstations. The measurements for geometry and the mural enhancement score were all analyzed at the same time in a single session. Locations of measurements were in the parietal and in the frontal branch of the superficial temporal artery within a range of $3-\mathrm{cm}$ length, $2-5 \mathrm{~cm}$ distal of the bifurcation. Wall thickness measurements of $>0.6 \mathrm{~mm}$ were considered pathologic. Measurements of the superficial occipital artery were performed at the level of the confluence sinus, which was readily revealed in each transversal image. The occipital arteries were evaluated according to the same criteria as the superficial temporal artery. For subjects scored as GCA positive by the MR examination, the vessel parameters of the diseased vessel branch were documented, whereas for the GCA-negative cases, the average values of both sides were used in the analysis. In addition, the MR score was reported by 2 individual radiologists to evaluate observer agreement of the MR diagnosis.

\section{Statistical Analysis}

The observer agreement was evaluated with a Cohen $\kappa$ test based on the MR diagnosis for GCA. The sensitivity and specificity of the MR 


\begin{tabular}{|c|c|c|c|c|c|c|c|c|c|}
\hline Comparison & $n$ & TP & TN & FP & FN & Sens & Spec & PPV & NPV \\
\hline MR vs ACR (all patients) & 64 & 25 & 32 & 1 & 6 & 80.6 & 97.0 & 96.2 & 84.2 \\
\hline MR vs ACR (<10 days of steroids) & 50 & 24 & 21 & 1 & 4 & 85.7 & 95.5 & 96.0 & 84.0 \\
\hline MR vs $A C R$ ( $>10$ days) & 14 & 1 & 11 & 0 & 2 & 33.3 & 100 & 100 & 84.6 \\
\hline Histo vs ACR & 32 & 21 & 5 & 0 & 6 & 77.8 & 100 & 100 & 45.5 \\
\hline MR vs ACR (patients with histo) & 32 & 22 & 5 & 0 & 5 & 81.5 & 100 & 100 & 50.0 \\
\hline MR vs histo & 32 & 19 & 8 & 3 & 2 & 90.5 & 72.7 & 86.4 & 80.0 \\
\hline Wall thickness vs ACR (all patients) & 64 & 22 & 26 & 7 & 9 & 71.0 & 78.8 & 75.9 & 74.3 \\
\hline
\end{tabular}

Note:-Results of the MR evaluation and temporal artery biopsy in the diagnosis of giant cell arteritis (GCA). The number of subjects (n), true-positive (TP), true-negative (TN), false-positive (FP), and false-negative (FN) cases, as well as the values for sensitivity (Sens), specificity (Spec), positive predictive value (PPV), and negative predictive value (NPV) are reported for all of the patients in the study and various subgroups. Histo indicates histology; ACR, American College of Rheumatology.

\begin{tabular}{|c|c|c|c|c|c|c|c|}
\hline GCA & Subjects, $n$ & CRP, $\mathrm{mg} / \mathrm{dL}$ & ESR & Wall, mm & Lumen, mm & Lumen/wall & MR score \\
\hline Positive & 31 & $11.2 \pm 7.1$ & $76.1 \pm 30.9$ & $0.74 \pm 0.32$ & $0.65 \pm 0.38$ & $1.23 \pm 1.1$ & $2.03 \pm 1.05$ \\
\hline Negative & 33 & $7.05 \pm 7.3$ & $48.8 \pm 32.3$ & $0.39 \pm 0.18$ & $0.84 \pm 0.29$ & $2.63 \pm 1.43$ & $0.45 \pm 0.56$ \\
\hline$P$ value & - & 0.033 & 0.0024 & 0.00014 & 0.036 & 0.000676 & 0.000009 \\
\hline$P$ value ranking & - & 5 & 4 & 2 & 6 & 3 & 1 \\
\hline
\end{tabular}

Note:-Various parameters for the GCA-positive and GCA-negative patient collectives. GCA indicates giant cell arteritis. The C-reactive protein (CRP), erythrocyte sedimentation rate (ESR), wall thickness, lumen diameter, ratio of lumen and wall, and the MR mural inflammation score are presented as mean \pm SD. The bottom row shows the ranking of their $P$ values when used as a single predictor for the ACR-based diagnosis, where 1 represents the lowest $P$ value and 6 the highest.

evaluation of mural inflammation was calculated for all of the patients, for the subgroups that had received corticosteroid treatment for less than 10 days, the subgroup that had received steroids for 10 days or more, and for the subgroup of 32 patients who had undergone a TAB. The sensitivity and specificity of the histologic examination were also evaluated for the patients who had undergone a TAB and were compared with the MR score.

The mean values and SDs for age, CRP, ESR, wall thickness, and lumen diameter; the ratio of lumen diameter to wall thickness; and the MR evaluation score were calculated for both patient groups. These parameters were tested for their suitability to act as surrogates for the ACR diagnostic criteria. Because these measurements are likely to be correlated, they were first assessed individually. A logistic regression model was fit separately to each of the potential predictors to rank their ability in predicting the ACR diagnosis. The ranking was based on the $P$ value from the test of the null hypothesis that the coefficient of the predictor is 0 . In addition, a stepwise model selection procedure based on Akaike Information Criterion (AIC) was used to identify an optimal logistic regression model among all of the predictors. Stepwise model selection with AIC is a procedure for selecting the model with the best predictive power among the models under consideration. Of the models considered, the model with the lowest value of AIC is selected as the final model. We performed backward stepwise selection by using the step procedure in the $R$ statistical environment. ${ }^{14}$ This procedure was applied to a model with the following predictors: CRP, ESR, wall thickness, lumen diameter, ratio of lumen diameter to wall thickness, and MR evaluation score. The step procedure removed terms from the model with the goal of minimizing AIC. The predictors in the AIC-selected model were tested for statistical significance.

\section{Results}

In all of the patients, the superficial cranial arteries could be successfully evaluated. The numbers of true-positive, truenegative, false-positive, and false-negative outcomes for the MR evaluation of mural inflammation and histology and the resulting sensitivities, specificities, positive predictive values, and negative predictive values are summarized in Table 1. For all 64 of the patients, MR had a sensitivity of $80.6 \%$ and a specificity of $97.0 \%$. If only the 50 patients who had received corticosteroid treatment for less than 10 days are included in the analysis, then the sensitivity of MR is $85.7 \%$ and the specificity is $95.5 \%$. For the 14 patients who had received steroids for 10 days or more, the sensitivity and specificity were $33.3 \%$ and $100 \%$, respectively. Observer agreement tested with a Cohen $\kappa$ test based on the MR diagnosis for GCA demonstrated a substantial strength of agreement with a $\kappa$ of 0.68 and a confidence interval of $95 \%$ from 0.50 to 0.86 .

Thirty two of the patients also had a unilateral biopsy of the temporal artery, and the sensitivity of the biopsy was $77.8 \%$ and the specificity was $100 \%$. TAB was false-negative in 6 cases, in 3 of which MR imaging was negative as well. In 1 patient, the occipital arteries were inflamed with spared temporal arteries while the TAB was taken from the right temporal artery. In the other 2 patients, the right and left temporal arteries showed signs of mural inflammation, respectively. Negative biopsy of the temporal artery was presumably because of skip lesions.

The mean values and SDs for the parameters measured in both patients groups are shown in Table 2. Patients who were GCA positive had a mean value for ESR of $76.1 \mathrm{mg} / \mathrm{dL}( \pm 30.9$ $\mathrm{mg} / \mathrm{dL})$ and for CRP of $11.2 \mathrm{mg} / \mathrm{dL}( \pm 7.1 \mathrm{mg} / \mathrm{dL})$. For the patients in the control group, the mean value for ESR was 48.8 $\mathrm{mg} / \mathrm{dL}( \pm 32.3 \mathrm{mg} / \mathrm{dL})$ and $7.05 \mathrm{mg} / \mathrm{dL}( \pm 7.3 \mathrm{mg} / \mathrm{dL})$ for CRP. The mean of the enhanced wall thickness increased from $0.39 \mathrm{~mm}( \pm 0.18 \mathrm{~mm})$ to $0.74 \mathrm{~mm}( \pm 0.32 \mathrm{~mm})$, and the lumen diameter decreased from $0.84 \mathrm{~mm}( \pm 0.29 \mathrm{~mm})$ to 0.65 $\mathrm{mm}( \pm 0.38 \mathrm{~mm})$ for the patients who were GCA positive. Accordingly, the mean lumen-to-wall ratio also decreased from $2.63( \pm 1.43)$ to $1.23( \pm 1.1)$. The mean MR score increased from $0.45( \pm 0.56)$ to $2.03( \pm 1.05)$ for the patients who were GCA positive. Based on the logistic regression models of ACR for each predictor, the following order (most to least predictive) was established: MR score, wall thickness, ratio of wall thickness to lumen diameter, ESR, CRP, and lumen diameter. All of these predictors were found to be statistically significant $(P<.05)$, and these results indicate that the MR score and the wall measurements are good predictors and con- 


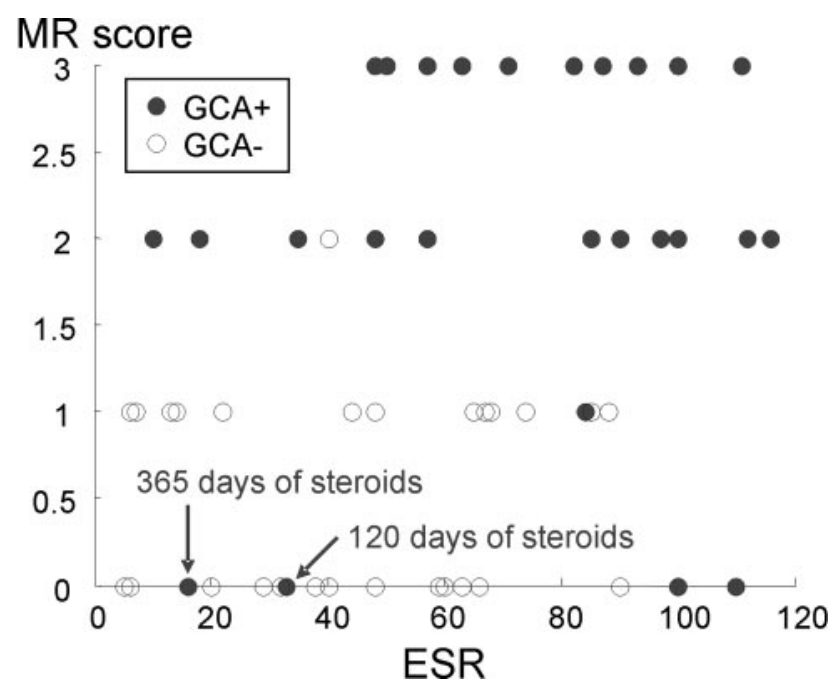

Fig 2. Feature plot MR score of mural inflammation versus ESR. Patients with an elevated ESR and a high MR score are all diagnosed GCA positive according to the ACR criteria. Patients with a low ESR and a low MR score are mostly diagnosed GCA negative. Please note that 2 of the false-negative MR findings with a very low MR score are imaged after long treatment with corticosteroids. Single points in the plot may represent $>1$ patient in case of identical values.

firm that elevated ESR is an important indicator for GCA. The histology results were excluded from this part of the statistical analysis because biopsies were only performed on half of all patients with a bias toward subjects who were GCA positive.

The stepwise selection procedure resulted in the following model with 2 parameters, the MR score for mural inflammation, $\chi_{1}$, and ESR, $\chi_{2}$, to predict the probability, $\hat{P}$, that the ACR diagnosis is GCA:

$$
\mu=\hat{\beta}_{0}+\chi_{1} \hat{\beta}_{1}+\chi_{2} \hat{\beta}_{2} \text {, with } \hat{P}=\frac{e^{\mu}}{1+e^{\mu}}
$$

The intercept $\beta_{0}$ was found to be -3.75 , and the scaling coefficients $\beta_{1}$ and $\beta_{2}$ were 1.83 and 0.026 . This logistic regression model demonstrated statistical significance for the MR score $(P=.000045)$ and the ESR $(P=.032)$. Figure 2 demonstrates the distribution of GCA-positive and -negative subjects as a function of their ESR and MR score. Although the MR score separates the GCA-positive and -negative subjects pretty well, the ESR further assists in defining a cluster of the GCA-positive subjects. AIC-based model selection was used a second time with all of the previous predictors except the MR score, because this parameter is somewhat subjective based on the evaluation of the radiologist. In this scenario, a model with the 2 parameters wall thickness $(P=.00038)$ and $\operatorname{ESR}(P=.024)$ was found to be optimal with an only slightly decreased confidence compared with the model with MR score and ESR. Wall thickness may have not been initially selected, because it is probably correlated with MR score.

\section{Discussion}

Visualization of the cranial arteries was well feasible with the use of this noninvasive approach of high-resolution MR imaging. It contributed to the diagnosis of GCA, because mural inflammatory changes could be successfully identified in affected arteries and differentiated from unaffected arteries. High values for sensitivity and specificity, $80.6 \%$ and $97.0 \%$, respectively, were found compared with the final rheumatologist's diagnosis, including the clinical criteria of the ACR, $\mathrm{TAB}$ if available, and follow-up examinations. The sensitivity increased to $85.5 \%$ in those 50 patients who were investigated within 10 days after the start of corticosteroid treatment. Furthermore, the cranial involvement pattern could be assessed. This is of particular interest, because GCA is known to affect arteries intermittently rather than continuously. ${ }^{15,16}$ Falsenegative biopsy results may occur if biopsy specimens are taken from an unaffected segment of the temporal artery. ${ }^{17,18}$ Two false-positives in the comparison of MR versus histology (Table 1) had mural inflammatory changes in MR imaging, but histology revealed no signs of GCA. Formally, they have to be counted as false-positive in this particular comparison. However, presumably because of sample error (skip lesions in GCA), a noninflamed segment was harvested in the TAB. In those 2 patients, the rheumatologist made the diagnosis of GCA by clinical means despite the negative biopsy. Therefore, MR imaging was actually true-positive, and histology was false-negative, as it is reflected in the first row of Table 1, MR imaging versus ACR, which includes all of the clinical, serologic, and histologic results for the final rheumatolgist's diagnosis. This actually shows the great benefit of high-resolution MR imaging, because it depicts the entire cranial circumference simultaneously and allows us to assess the inflammatory involvement pattern. Potentially, the number of false-negative biopsies can be reduced if the cranial involvement pattern is known, and TAB can be performed in the most inflamed segment rather than performing a standard procedure (Fig 3 ).

The statistical analysis showed that the combination of the mural inflammation score, as determined on a 4-point scale, and the ESR value produce the best model to predict the ACR diagnosis. The nonsubjective and quantitative wall thickness is also a powerful predictor and can replace the somewhat more subjective inflammation score. MR imaging of the superficial cranial arteries has been shown to be feasible at $1.5 \mathrm{~T} .{ }^{13}$ However, using the higher SNR at 3T renders superior image quality compared with $1.5 \mathrm{~T}$.

TAB can be positive up to 2 weeks after steroid therapy. ${ }^{19}$ However, steroid therapy leads to decreased enhancement in large-vessel vasculitis. ${ }^{20-22}$ In this study, 3 patients were diagnosed as GCA positive and were imaged after more than 10 days of corticosteroid treatment. One was diagnosed correctly with MR imaging (14 days of treatment), whereas the other 2 subjects ( 3 months and 12 months of treatment) did not show any signs of mural inflammation. On the other hand, the 9 patients who were GCA negative who received corticosteroid treatment for more than 10 days before the MR examination were all correctly diagnosed. It is uncertain after how many days of high-dose steroids MR imaging becomes false-negative. However, because irreversible visual loss is a dreaded complication of GCA, corticosteroid therapy needs immediate initiation. Potentially, some of the false-negative MR imaging findings were due to steroid effects on mural enhancement, because 2 of these patients received steroids for more than 3 months before the MR examination.

Duplex ultrasonography investigations give information on vessel wall and halo, as well as on blood flow. ${ }^{5,7}$ It is a relatively accurate test for diagnosing GCA with sensitivities of $73 \%-93 \%$ and specificities of $89 \%-93 \% .^{5,7}$ With the pre- 

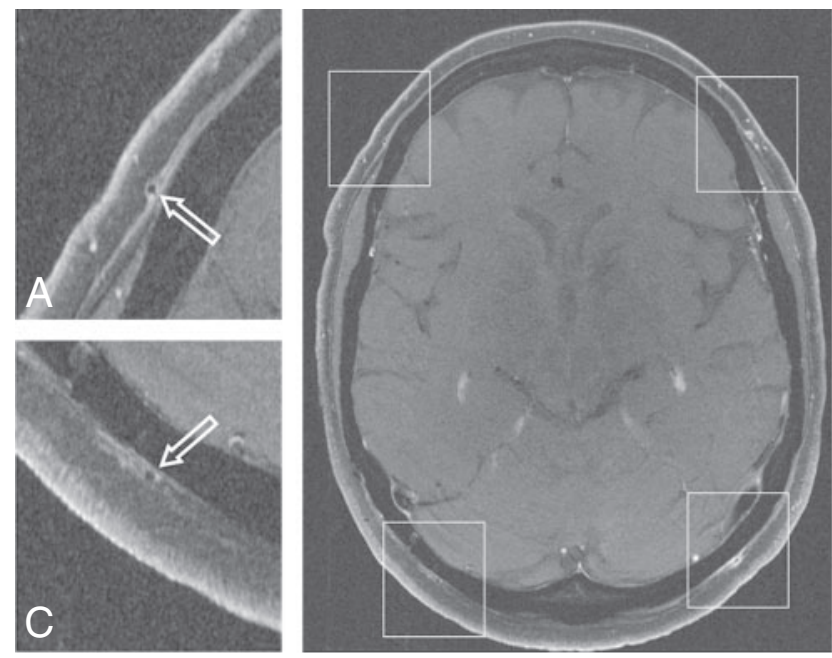
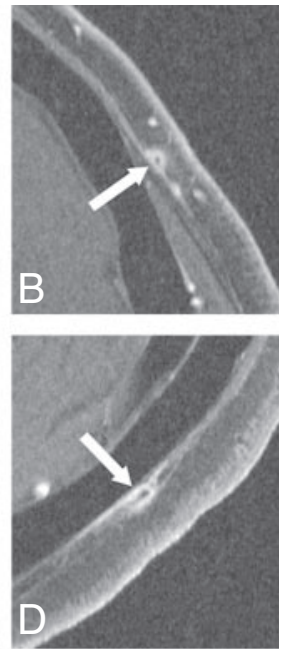

Fig 3. $3 T$ transversal contrast fat-suppressed T1-weighted SE image acquired with the large FOV that covers the entire cranial circumference. Enlargements of the temporal branch of the superficial temporal arteries $(A$ and $B)$ and of the superficial occipital arteries $(C$ and $D)$ demonstrate the cranial involvement pattern. Mural thickening and inflammatory changes are depicted in the left temporal artery (enlargement $B, 0.7-\mathrm{mm}$ mural thickness, rated as " 3 ") and occipital artery (enlargement $D, 0.7-\mathrm{mm}$ mural thickness, rated as " 3 "), whereas the right-sided arteries display no signs of mural inflammation (enlargements $A$ and $C, 0.2-\mathrm{mm}$ mural thickness, both rated as " 0 "). Temporal artery biopsy validates GCA in this patient. sented MR imaging protocol, comparably high spatial resolution could be achieved. However, ultrasonography is observer dependent and shows various sensitivities depending on the pretest disease prevalence in the different study populations. ${ }^{11}$ Concerning high-resolution MR imaging of the superficial cranial arteries, only limited experience exists today. To investigate the value of the 2 noninvasive imaging modalities, we have initiated a prospective multicenter trial comparing the diagnostic performance of ultrasonography with high-resolution MR imaging.

PET with $\left[{ }^{18} \mathrm{~F}\right]$ fluoro-2-deoxy-D-glucose is very sensitive in detecting extracranial involvement of large vessel vasculitis. ${ }^{23}$ However, it provides no information on inflammatory changes of the superficial cranial arteries, because the spatial resolution and sensitivity of positron-emission tomography are not sufficient to evaluate vessels with a size smaller than $2 \mathrm{~mm}$.

When interpreting the presented data, a certain referral bias has to be taken into account: referral by experienced rheumatologists, ophthalmologists, or neurologists may lead to a high pretest probability for positive GCA. However, patients with a small likelihood of GCA were also referred for MR imaging for exclusion of mural contrast enhancement. In the end, there was an almost equal distribution of patients with GCA and patients in whom GCA was ruled out. Ideally, this study would have 2 well-demarcated groups: one with biopsyproved GCA fulfilling the ACR criteria and one age- and gender-matched group without any features of GCA. However, by recruiting the control subjects from the patients who initially were suspected of having GCA but finally were diagnosed as not having GCA, our study setting comes closer to the daily routine that confronts clinicians.

A TAB would have been desirable in every patient in this study, but histologic proof of GCA was achieved for only 32 patients. However, TAB was performed in 27 of the 31 patients with the final diagnosis of GCA. Of the 33 patients not fulfilling the ACR criteria, biopsies were performed in only 5 subjects, all with negative results. In the other 28 patients, GCA was ruled out on a clinical basis.

For statistical purposes, large numbers of patients are favorable. To gain higher statistic power, we included patients who were investigated during our initial experience with this novel MR imaging approach. ${ }^{3,13}$
A future approach to improve specificity could be using "intelligent" contrast agents that specifically bind to substances that typically or even uniquely occur in GCA. If such a contrast agent could be developed and its sensitivity and specificity could be proved, TAB would no longer be necessary.

\section{Conclusion}

This study shows that high-resolution MR imaging allows for the detailed visualization of the temporal arteries and the occipital arteries bilaterally. Corticosteroid treatment influences the diagnostic accuracy of MR imaging, and investigations should be performed before or soon after onset of treatment. Good agreement with histology and diagnosis according to the ACR criteria was found. With the proposed MR imaging protocol, GCA-positive and -negative cases can be separated, and ESR further assists in defining a cluster of the GCA-positive cases. With mural thickening and contrast enhancement as valuable vasculitic MR imaging findings, contrast-enhanced, high-resolution MR imaging is a valuable noninvasive imaging technique in the diagnosis of GCA. The data presented in this study demonstrate the potential of high-resolution MR imaging to eventually replace TAB.

\section{Acknowledgments}

We thank Jürgen Hennig and Peter Vaith for their continuing medical and technical advice and support.

\section{References}

1. Horton B, Magath T, Brown G. An undescribed form of arteritis of the temporal vessels. Proc Mayo Clin 1932;7:700-01

2. Salvarani C, Cantini F, Boiardi L, et al. Polymyalgia rheumatica and giant-cell arteritis. $N$ Engl J Med 2002;347:261-71

3. Bley TA, Weiben $\mathrm{O}$, Uhl M, et al. Assessment of the cranial involvement pattern of giant cell arteritis with 3T magnetic resonance imaging. Arthritis Rheum 2005;52:2470-77

4. Nuenninghoff DM, Hunder GG, Christianson TJ, et al. Incidence and predictors of large-artery complication (aortic aneurysm, aortic dissection, and/or large-artery stenosis) in patients with giant cell arteritis: a population-based study over 50 years. Arthritis Rheum 2003;48:3522-31

5. Pfadenhauer K, Weber H. Giant cell arteritis of the occipital arteries-a prospective color coded duplex sonography study in 78 patients. J Neurol 2003;250:844-49

6. Hunder GG, Bloch DA, Michel BA, et al. The American College of Rheumatology 1990 criteria for the classification of giant cell arteritis. Arthritis Rheum 1990;33:1122-28 
7. Schmidt WA, Kraft HE, Vorpahl K, et al. Color duplex ultrasonography in the diagnosis of temporal arteritis. $N$ Engl J Med 1997;337:1336-42

8. Weyand CM, Goronzy JJ. Medium- and large-vessel vasculitis. $N$ Engl J Med 2003;349:160-69

9. Reinhard M, Schmidt D, Hetzel A. Color-coded sonography in suspected temporal arteritis-experiences after 83 cases. Rheumatol Int 2004;24:340 - 46

10. Salvarani C, Silingardi M, Ghirarduzzi A, et al. Is duplex ultrasonography useful for the diagnosis of giant-cell arteritis? Ann Intern Med 2002;137:232-38

11. Karassa FB, Matsagas MI, Schmidt WA, et al. Meta-analysis: test performance of ultrasonography for giant-cell arteritis. Ann Intern Med 2005;142:359-69

12. Frayne R, Goodyear BG, Dickhoff $P$, et al. Magnetic resonance imaging at $\mathbf{3 . 0}$ Tesla: challenges and advantages in clinical neurological imaging. Invest $R a-$ diol 2003;38:385-402

13. Bley TA, Wieben $\mathrm{O}$, Uhl M, et al. High-resolution MRI in giant cell arteritis: imaging of the wall of the superficial temporal artery. AJR Am J Roentgenol 2005; 184:283-87

14. Maindonald JB, Braun WJ. Data Analysis and Graphics Using R. Cambridge: Cambridge University Press; 2003

15. Klein RG, Campbell RJ, Hunder GG, et al. Skip lesions in temporal arteritis. Mayo Clin Proc 1976;51:504-10
16. Hall S, Persellin S, Lie JT, et al. The therapeutic impact of temporal artery biopsy. Lancet 1983;2:1217-20

17. Albert DM, Ruchman MC, Keltner JL. Skip areas in temporal arteritis. Arch Ophthalmol 1976;94:2072-77

18. Ashton-Key MR, Gallagher PJ. False-negative temporal artery biopsy. Am J Surg Pathol 1992;16:634-35

19. Achkar AA, Lie JT, Hunder GG, et al. How does previous corticosteroid treatment affect the biopsy findings in giant cell (temporal) arteritis? Ann Intern Med 1994;120:987-92

20. Bley TA, Warnatz K, Wieben O, et al. High-resolution MRI in giant cell arteritis with multiple inflammatory stenoses in both calves. Rheumatology (Oxford) 2005;44:954-55

21. Bley TA, Wieben O, Leupold J, et al. MRI findings in temporal arteritis. Circulation 2005; 111:e260

22. Choe YH, Kim DK, Koh EM, et al. Takayasu arteritis: diagnosis with MR imaging and MR angiography in acute and chronic active stages. J Magn Reson Imaging 1999;10:751-57

23. Walter MA, Melzer RA, Schindler C, et al. The value of [18F]FDG-PET in the diagnosis of large-vessel vasculitis and the assessment of activity and extent of disease. Eur J Nucl Med Mol Imaging 2005;32:674-81 